\title{
Low-Speed and Suspended Propagation of Light Pulses in Atomic Medium with Electromagnetically Induced Transparency
}

\author{
D. Dziczek, B. Ziȩtek And S. ChWirot \\ Department of Atomic, Molecular and Optical Physics \\ Institute of Physics, Nicolaus Copernicus University \\ Grudziądzka 5/7, 87-100 Toruń, Poland
}

(Received May 18, 2004)

\begin{abstract}
Experiments on hot rubidium vapor as an atomic medium with electromagnetically induced transparency for radiation of $795 \mathrm{~nm}$ wavelength (D1 absorption line of rubidium) are reported. Propagation of light pulses with velocity reduced to $11.5 \mathrm{~km} / \mathrm{s}$ has been observed with a significant modification of the shape of square input pulses to adapt to the spectral window of induced transparency. So-called light storage, i.e. suspension of the propagation of light pulses in the medium by temporary turn-off of the electromagnetically induced transparency state was also demonstrated. "Storage" periods of several microseconds, limited by relatively fast relaxation of atomic spin polarization in the volume of interaction of atoms with radiation, were obtained.
\end{abstract}

PACS numbers: 32.80.Qk, 42.25.Bs, 42.50.Gy

\section{Introduction}

An interaction of coherent radiation with matter in the conditions of resonant optical transitions receives continuous research interest because of vast amounts of data provided on the structure of matter and numerous applications in science and technology. Recently, a significant part of the interest has been focused on dramatic modifications induced by a strong resonant laser beam in the optical properties of an atomic medium probed by a second weaker beam. Such modifications are related to the phenomenon of coherent population trapping (CPT) 
first observed by Alzetta et al. [1] and interpreted by Arimondo and Oriols [2] as a partial confinement of the atomic population within a special (coherent) superposition of two atomic states, both radiatively coupled to a third one (see also [3-5] and a review [6]). One of the consequences of the presence of the saturating beam can be a reduction of the absorption of the probe beam by many orders of magnitude - the state of electromagnetically induced transparency (EIT) of the medium [7-9]. In such cases, a very steep dispersion characteristic of extremely narrow EIT resonances results in low group velocities of propagation of probe beam pulses [10-12]. In an ultracold atomic gas (Bose-Einstein condensate of sodium atoms) the velocity as low as $17 \mathrm{~m} / \mathrm{s}$ has been observed [13].

The probe beam pulse velocity depends on the intensity of the laser beam which induces the transparency $[10,13]$, so it can be controlled externally (for this reason the beam is commonly named the control beam) and even reduced to zero by turning the beam off completely. The propagation of light pulses in the EIT media is accompanied by co-propagating waves of varying collective polarization or excitation of atoms involved in CPT, termed dark-state polaritons [14]. A reduction of the velocity of propagation to zero, by extinguishing the control beam, effectively "freezes" the specific state of the atomic ensemble holding the information on light which has been "trapped" this way.

Even more fascinating is the fact that this process is reversible. Turning the control beam back on allows a retrieval of the information imprinted in the state of atomic ensemble, a recreation of the probe beam pulses, and resuming their propagation in the medium. This has been demonstrated experimentally by Liu et al. [15] in a cloud of cold sodium atoms and by Phillips et al. [16] in the hot rubidium vapor. Those two experiments have proved that it is possible to "map" the state of photon ensemble onto spatial distribution of excitation or polarization in the atomic system, to "store" it for some period of time and then to retrieve it. The idea of influencing the state of the atomic ensemble during the "storage" phase of this sequence follows naturally. Besides the new method of coherent control over the state of atoms with help of light, a new way of manipulating the state of light by means of atoms should be possible. The problems of these new and promising techniques of modern optics are presently under extensive investigations (some of them have been reviewed by Lukin in [17], see also Dutton et al. [18]), including the "storage" of light in non-classical quantum state [19].

We report results of an introductory experiment on suspending the propagation of light pulses in rubidium vapor and their subsequent regeneration and release from the medium. The idea of the experiment is, in principle, the same as in the first impressive demonstrations of the effect $[15,16]$. The EIT state was induced in the atomic medium consisting of ${ }^{87} \mathrm{Rb}$ atoms — as in experiment of Phillips et al. [16]. Also the choice of the hyperfine levels involved in the CPT was the same. However, the cell contained also the second isotope of rubidium (atoms were in the proportions of the natural mixture: ${ }^{85} \mathrm{Rb}-72.17 \%,{ }^{87} \mathrm{Rb}-27.83 \%$ ) 
and there was no buffer gas in the vapor cell. This allowed the observation of the strong and destructive effects of the relaxation of atomic polarization on the information on light pulses imprinted in the atomic system while their propagation was stopped.

We used probe and control beams operated independently which gave us a little more flexibility in timing relations between laser pulses compared to the experimental setup described in [16], although at the expense of beam alignment problems.

We present results of the experiments in which we used square input pulses, demonstrating the extend of pulse shape modifications caused by the EIT medium to accommodate them for propagation within the spectral window of induced transparency (the dynamics of establishing EIT based on theoretical simulations for a cold atomic sample was discussed in [20]). Only the shape which can be "admitted" by the medium can be imprinted in the spatial distribution of the atomic polarization when the propagation is suspended. This condition determines the form of information on light which can be "stored" this way.

\section{Apparatus}

A simplified schematic of the experimental setup is presented in Fig. 1. The cylindrical rubidium cell made of Pyrex glass (diameter $25 \mathrm{~mm}$, length $50 \mathrm{~mm}$ ) was housed in an aluminum block heated by circulation of hot oil. The temperature of the block was maintained constant (within $1^{\circ} \mathrm{C}$ ) during the entire experimental session. The heater was surrounded by a two-layer magnetic shield (CO-NETIC alloy, Magnetic Shields Corp.) reducing the influence of ambient magnetic field on rubidium atoms. A cylindrical coil was located inside the shield to compensate the residual magnetic field and provide axial test field used for EIT state diagnostics. The elements of the heating housing are not shown in Fig. 1 to simplify the drawing. The cell contained the natural mixture of rubidium isotopes. No buffer gas was used and there was no internal coating of the glass to slow down the relaxation of atomic polarization by atom-atom and atom-wall collisions. The concentration of rubidium atoms was approximately $1 \times 10^{13}$ atoms $/ \mathrm{cm}^{3}$ (the estimate based on the cell temperature of $100^{\circ} \mathrm{C}$ ). The corresponding concentration of ${ }^{87} \mathrm{Rb}$ atoms, the EIT medium was composed of, was approximately $2.8 \times 10^{12}$ atoms $/ \mathrm{cm}^{3}$.

Multiple $\Lambda$-systems of atomic energy levels, shown in Fig. 2, consisted of Zeeman sublevels of the ground state $5{ }^{2} S_{1 / 2}$ level with the total angular momentum $F=2$ (bottom levels) and Zeeman sublevels of the excited $5^{2} P_{1 / 2}$ state with the total angular momentum $F=1$ (upper levels). Generally, each of the three sets of levels forming $\Lambda$-systems could be involved in the interaction with laser beams. The optical pumping of the atomic ensemble by the relatively strong control laser beam should cause some domination of one of the side $\Lambda$-systems. However depolarization due to collisions, clearly observed in the conditions of the presented experiment, prevented a complete trapping of the population of the atoms interacting 


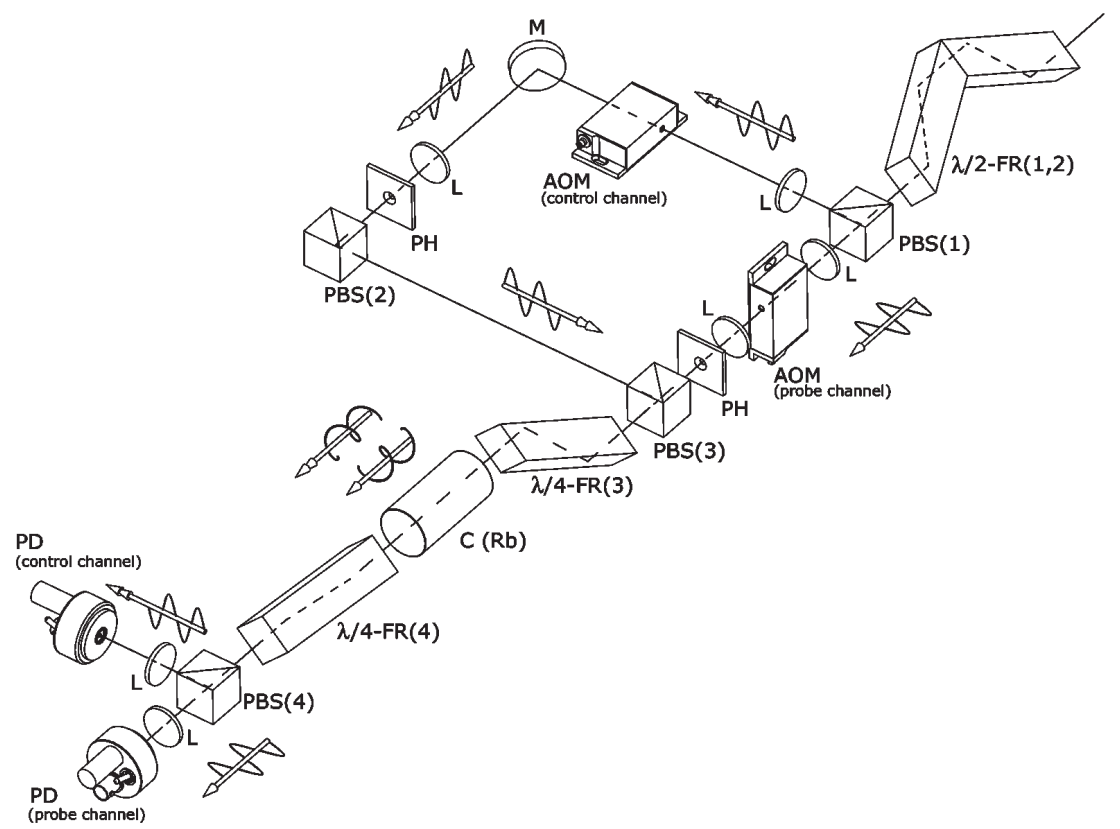

Fig. 1. Schematic of the experimental setup. C (Rb) - rubidium vapor cell, FR - Fresnel rhomb, PBS - polarizing beam splitter, L - lens, M - mirror, AOM - acousto-optic modulator, $\mathrm{PH}$ - pinhole, PD - photodiode detector. Arrows indicate directions and polarization of the laser beams.

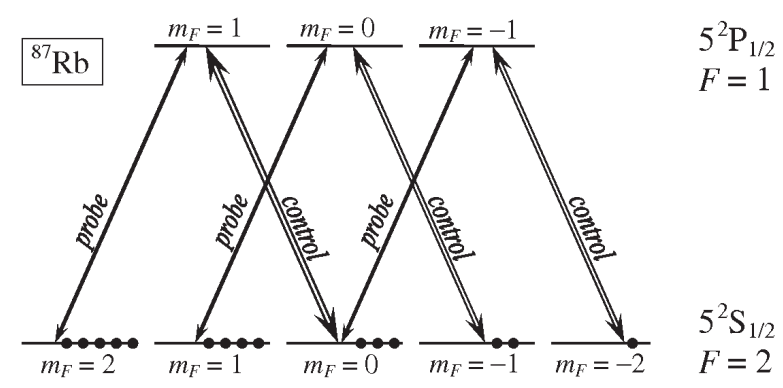

Fig. 2. Set of ${ }^{87} \mathrm{Rb}$ atom energy levels involved in the interaction with probe and control laser beams radiation in the absence of magnetic field. All three $\Lambda$-systems of levels contribute to the EIT state of the medium in the conditions of our experiment: atom-atom and atom-cell wall collisions counteract the optical pumping of atoms and trapping their population in one of the side $\Lambda$-systems, but a stronger control beam causes a non-uniform population of levels indicated schematically with circles at the bottom levels. 
with radiation within a single three-level system. Collision-induced depolarization affects the creation of the EIT state of rubidium vapor and, much stronger, the process of controlled suspension of the propagation of light pulses in the medium. The time of complete relaxation of the atomic ensemble, which was relatively short in the conditions of our experiment, limited the maximum time of the "storage".

Because of the degeneracy in energy (in the absence of magnetic field), the bottom levels of the $\Lambda$-systems could be coupled with the upper states by probe and control radiation derived from a single laser source. We used the output beam of MBR-110 Ti:Shapphire ring laser (Coherent, Inc.) tuned to the transitions between the mentioned levels, which comprise the hyperfine component of the D1 line of rubidium $(795 \mathrm{~nm})$ of the longest wavelength. The attenuated beam was divided with a polarizing beam-splitter cube into two beams with perpendicular planes of polarization. The transmitted part, polarized in horizontal plane was used as the probe beam and the reflected part, polarized in vertical plane, as the control beam. Relative intensities of the beams could be adjusted with a half-wave retarder (consisting of two coupled Fresnel rhombs). Two acousto-optic modulators $(\mathrm{AOM})$ operated in the binary mode $(O N / O F F)$ were used to independently control both probe and control channels. The beams deflected by the AOMs in the $O N$ state entered the next elements of the optical system via pinholes which blocked the transmitted direct beams. The sets of lenses used to focus the beams onto the crystals of the AOMs and then to collimate the emerging beams were also used to adjust their diameters. The diameter of the probe beam was $2 \mathrm{~mm}$ while the control beam, of slightly elliptical cross-section, was wider: $5 \mathrm{~mm}$ by $4 \mathrm{~mm}$. The control beam was directed with a mirror and a second polarizing beam-splitter cube onto a third one, which reflected it, so it overlapped the probe beam along its axis. The combined beams entered a Fresnel rhomb quarter-wave retarder, which converted their perpendicular linear polarizations into circular ones of opposite helicities. The total input powers of the probe and control beams interacting with ${ }^{87} \mathrm{Rb}$ atoms in the cell were $200 \mu \mathrm{W}\left(6.4 \mathrm{~mW} / \mathrm{cm}^{2}\right)$ and $7.8 \mathrm{~mW}$ $\left(49.7 \mathrm{~mW} / \mathrm{cm}^{2}\right)$, respectively. Circular polarizations of the beams transmitted by the atomic medium were subsequently converted back to linear ones (with perpendicular planes of polarization) by the next Fresnel rhomb $\lambda / 4$-retarder. The fourth polarizing beam-splitter cube was used to separate probe and control beams to allow independent measurements of their intensities with two fast photodiode detectors (DET210, Thorlabs) connected to a digital oscilloscope. Data acquisition was synchronized by signals from a programmable timing circuit, which was also providing two square waveforms for turning $O N$ and $O F F$ the AOMs in the probe and control channels. The rise and fall times of the signals of the photodiodes receiving beams toggled this way did not exceed $100 \mathrm{~ns}$. Relative positions of rising and falling edges of the signals controlling modulators as well as durations of beam $O N$ and $O F F$ periods in both channels could be adjusted in wide ranges with 50 ns increments. 


\section{Experimental procedure}

After stabilization of the operating temperature of the rubidium cell, the intensity of the probe beam was adjusted, so it was completely absorbed and dissipated by atoms (both in the steady state and the pulsing mode). The EIT state of the atomic medium was then optimized using the scanning of the test magnetic field and adjusting the polarizing optics and the overlap of the beams. After those preparatory stages the propagation of light pulses in the medium was tested.

In the main part of the experiment, the control laser beam was repeatedly turned off during the propagation of probe beam pulses in rubidium vapor to stop them in the medium for variable periods of time and then to regenerate them and resume their propagation by turning the control beam back on. We were recording averages of 200 "stored" probe beam pulses in one memory channel of the digital oscilloscope. Another memory channel was used to record corresponding signals from the control beam photodiode detector. Background signals from both detectors (probe and control beam photodiode waveforms with the probe beam blocked) were also recorded as a part of each measurement cycle, as well as the averages of samples of 200 probe pulses transmitted with the control beam constantly in the $O N$ state, to serve as references for stopped pulses.

\section{Results and discussion}

Figure 3 shows the shape of the pulse transmitted by rubidium atoms in the EIT state (solid line, average of 200 pulses). Reference waveform (dashed line) represents the input pulse (it was registered in the same way, except detuning the laser far from the resonance). The significant change in the shape can be explained if one takes into account that not all spectral components of the input pulse with relatively fast edges could be contained within the induced transparency window of the medium. The EIT medium "adjusts" the shape of the input pulses to the form which is "suitable" for propagation. The final shape of transmitted pulses, their heights and widths are also affected by the finite time-of-flight effects and the relaxation processes (as examined in simulations reported by Cerboneschi et al. [21]).

In the conditions of the experiment, $4 \mu \mathrm{s}$ long pulses were delayed by approximately $4 \mu \mathrm{s}$, which corresponds to the pulse propagation velocity of $11.5 \mathrm{~km} / \mathrm{s}$. The leading slopes of reshaped pulses started to emerge from the atomic medium at about the time of the falling edges of the input pulses entered the cell. Thus nearly whole pulses were contained in the medium thanks to their spatial compression (from $1.2 \mathrm{~km}$ in free space to $46 \mathrm{~mm}$ of the length of the path through the medium).

Figures 4 and 5 present results of the experiment for the time of control beam turn-off periods of $3,5,7,9$, and 11 microseconds. Considering both the 


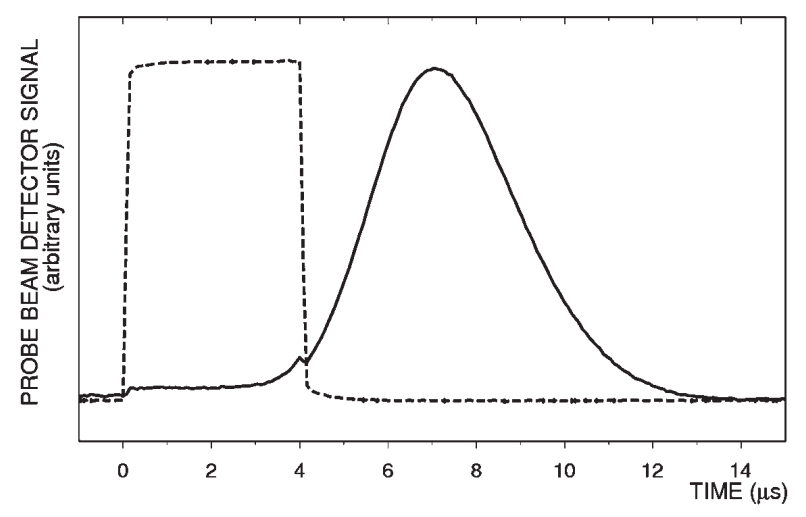

Fig. 3. Shape of probe beam pulses transmitted by rubidium vapor in the presence of the control beam electromagnetically inducing transparency of the medium. Solid line - an average of a sample of 200 transmitted pulses. Dashed line waveform represents the shape of input pulses - it was recorded in the same way except detuning of the laser far out of the resonance. Pulse height has been normalized to the transmitted pulse to illustrate a strong modification of the shape. The weak square pulse feature preceding the transmitted pulses is an artifact originating from the mutual "leaking" of the radiation between the probe and control channels due to imperfections of the polarizing optics.

height of stopped and regenerated pulses and their shape, we were able to successfully "store" pulses for times up to $9 \mu \mathrm{s}$. With the maximum light intensity decreasing with increasing time of suspension of the propagation (Fig. 4), the shape of transmitted pulses was quite well preserved as shown in Fig. 5. It is important to mention that the noticeable differences between the shapes of "stored" pulses and reference pulses reflect not only the effects of the "storage" and its imperfections. Reference pulses are averages of different samples of transmitted input signals. Though registered soon after "storage" sequences, they could have a slightly different shape due to instabilities in power of the laser beams.

For the suspension time of $11 \mu$ s the height of the transmitted pulse is less than 10 percent of the reference one, and its shape shows significant deformations. These deformations suggest that the pulse is an artifact of the operation of the background signal subtraction rather than stopped and regenerated probe beam pulse. So, in our experimental conditions, the pulses "stored" for more than approximately $10 \mu$ s could not be successfully re-created and released from the medium.

The existence of such limitations is expected in cases when hot atomic vapor is supposed to work as the medium in which the information on light pulses is imprinted in the polarization of a group of atoms - unavoidable relaxation of the polarization gradually reduces the number of atoms still "holding" the infor- 


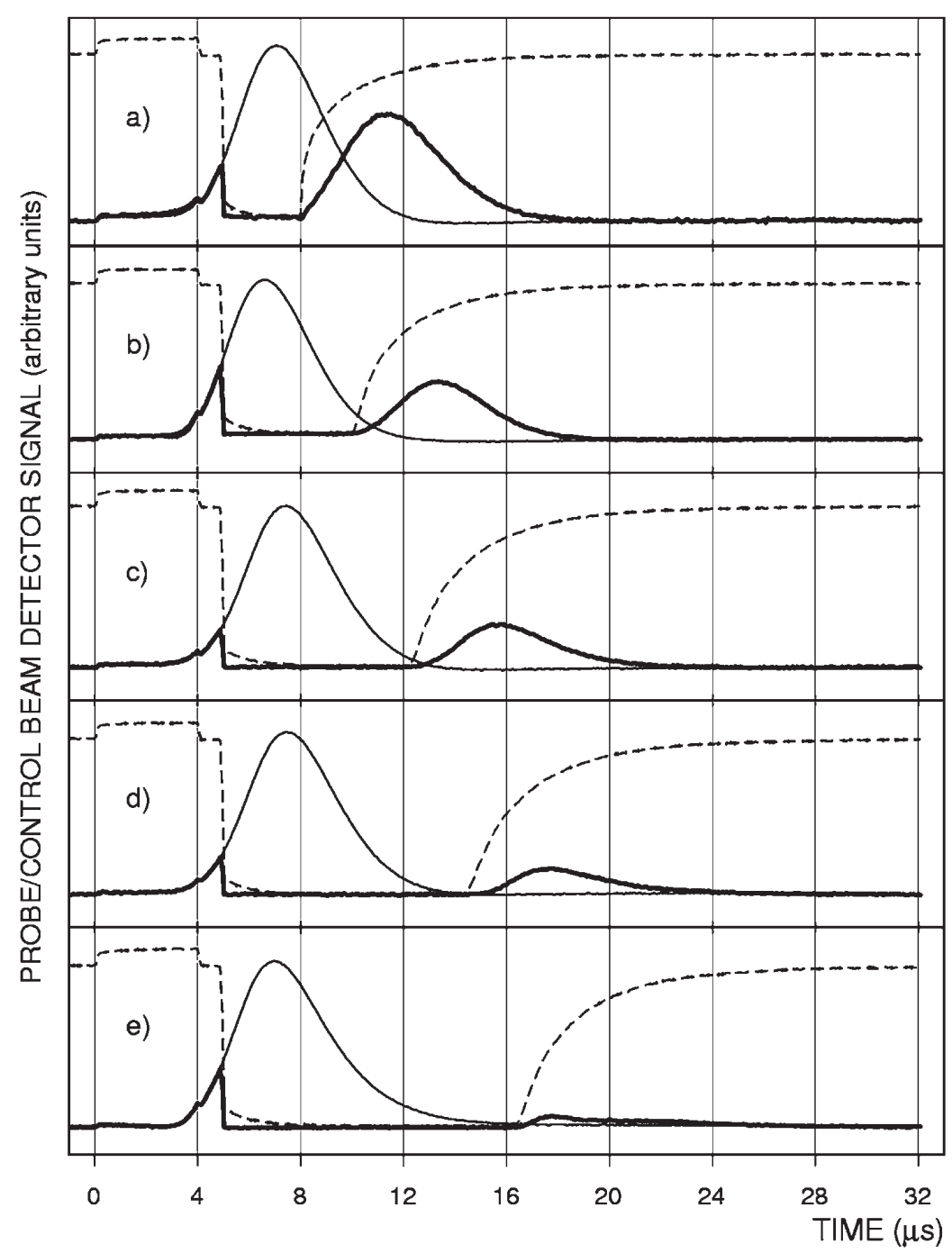

Fig. 4. Dependence of the height of pulses regenerated after suspension of their propagation in the EIT medium on the suspension time: (a) $3 \mu \mathrm{s}$, (b) $5 \mu \mathrm{s}$, (c) $7 \mu \mathrm{s}$, (d) $9 \mu \mathrm{s}$, and (e) $11 \mu \mathrm{s}$. Thick solid lines - stopped and recreated pulses (background from the control laser beam leaking to the probe detection channel subtracted), thin solid lines - reference waveform recorded with the control beam constantly in the $O N$ state; dashed lines - waveforms from the control beam detector (relatively weak square pulse feature preceding the $O F F$ state period of the control beam is from the probe beam radiation leaking to the control channel). The shape of the rising edge of this waveform indicates a relatively fast relaxation of polarization of the atoms in the conditions of our experiment, which limits the maximum pulse "storage" time. All waveforms are averages of samples of 200 cycles. 


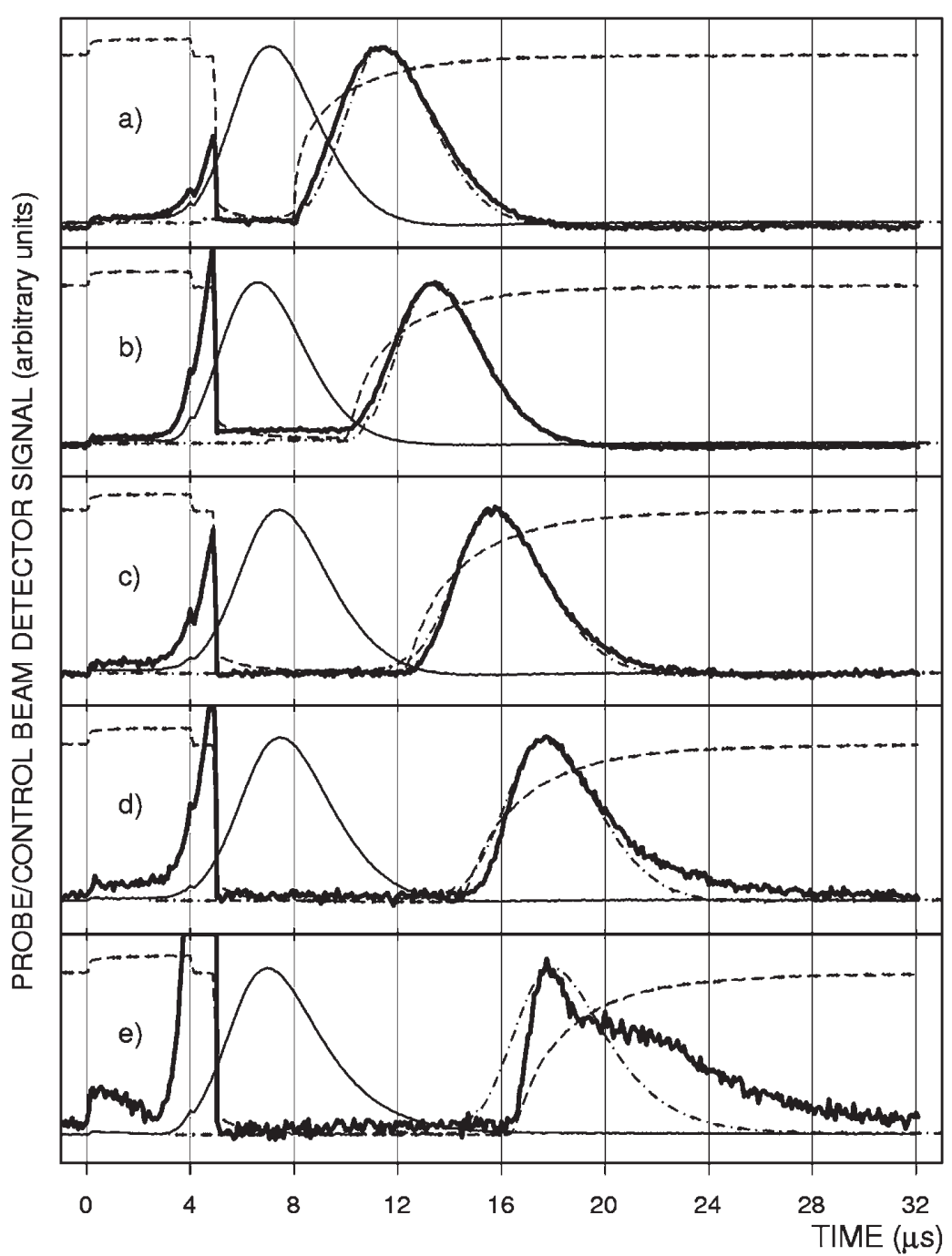

Fig. 5. Dependence of the shape of pulses regenerated after suspension of their propagation in the EIT medium on the suspension time: (a) $3 \mu \mathrm{s}$, (b) $5 \mu \mathrm{s}$, (c) $7 \mu \mathrm{s}$, (d) $9 \mu \mathrm{s}$, and (e) $11 \mu \mathrm{s}$. Line patterns as in Fig. 4. The waveforms representing stopped and recreated pulses were scaled to match the heights of the reference pulses. Additional waveforms (dash-dot lines) are the reference ones shifted in the way that their maxima coincide with the maxima of regenerated delayed pulses.

mation. However, in our case, the relaxation time and corresponding maximum "storage" time seems to be significantly shorter than one could expect, comparing for example with data reported in Ref. [16]. Nevertheless, there is a direct indication that the relaxation of atoms involved in studied processes is really fast in 
the conditions of our experiment. It clearly shows itself in the shape of the rising edges of the recorded signals from the control beam detector (included in all plots in Figs. 4 and 5 as dashed lines). If the majority of atoms remained in their polarization state during the "storage" time, the absorption of the light immediately after turning the control beam back on $\left(t_{\text {on }}\right)$ would be close to its level in the moment of turning it off $\left(t_{\text {off }}\right)$. As clearly seen in all presented waveforms, initial absorption at the moments $t_{\text {on }}$ was significantly higher than at $t_{\text {off }}$ and gradually decreased with time. In fact, the light was initially fully absorbed in all cases except the shortest "storage" time. The transmission was reaching values close to steady state saturation after approximately $8 \mu \mathrm{s}$ in the manner characteristic of optical pumping.

As already mentioned, fast relaxation can be explained by the fact that there was no buffer gas used to slow down depolarization caused by atom-atom and atom-wall collisions. Buffer gas would also reduce the number of polarized rubidium atoms escaping from the volume of interaction with laser beams (in average, at our operating temperature, atoms moving transversally to the direction of the beams could cross this volume in less than $20 \mu \mathrm{s}$ ).

Moreover, in excess of $72 \%$ of atoms in the vapor (isotope ${ }^{85} \mathrm{Rb}$ ) did not interact with the radiation and could not play the role of the "atomic memory", and at the same time could contribute to destruction of the polarization of the group of atoms involved in this interaction by atom-atom collisions. Estimations of the mean time between depolarizing collisions based on the electron exchange cross-section given in [22] yield values from $37 \mu$ s to $53 \mu$ s at our operating temperature. The combination with effects of other two mentioned mechanisms of depolarization of atoms in the interaction volume leads to a further reduction of the expected relaxation time.

An additional important factor strongly influenced the ability of the atomic ensemble to hold the information on light pulses and our ability to successfully notice it. The number of atoms belonging to the ensemble was very low because only a small fraction of ${ }^{87} \mathrm{Rb}$ atoms was involved in interaction with radiation. The laser source used, besides excellent tuning ability, is characterized by low spectral linewidth $(100 \mathrm{kHz}$, as specified by the manufacturer). It is extremely narrow compared with the Doppler-broadened linewidth of the transition under study (exceeding $500 \mathrm{MHz}$ at our operating temperature). Therefore, a very limited number of rubidium atoms was polarized by the control laser beam and propagating probe beam pulses. This number was quickly decreasing after the control beam was turned off as the result of strong relaxation processes. For the "storage" times of $11 \mu \mathrm{s}$ and longer, the remaining number of polarized atoms was not sufficient to recreate pulses strong enough to be extracted from recorded signals.

After a more detailed inspection of the shape of the regenerated pulses (Fig. 5) one can notice that their leading edges do not complement the portions of the pulses which were allowed to leave the cell before the control beam was shut off 
(they were considerably smaller than in experiment of Phillips et al. [16] in which the shapes of suspended and recreated portions of the pulses matched the shapes of unsuspended parts). This could be probably considered as a deformation of the fronts of the pulses resulting from the gradual increase in intensity of the control beam. However, the effects of the collisional relaxation and optical pumping of atoms underlaying the effect of suspension of propagation make the problem too complex to attempt a deeper interpretation within the scope of this report, which was intended mainly as a presentation of the experimental results.

\section{Acknowledgments}

This work was carried out at the National Laboratory for Atomic Molecular and Optical Physics in Torun, Poland and was supported by the State Committee for Scientific Research (grant No. PBZ KBN 043/P03/2001).

\section{References}

[1] G. Alzetta, A. Gozzini, L. Moi, G. Oriols, Nuovo Cimento B 36, 5 (1976).

[2] E. Arimondo, G. Oriols, Nuovo Cimento Lett. 17, 333 (1976).

[3] H.R. Gray, R.M. Whitley, C.R. Stroud, Opt. Lett. 3, 218 (1978).

[4] G. Alzetta, L. Moi, G. Oriols, Nuovo Cimento B 52, 209 (1979).

[5] G. Oriols, Nuovo Cimento B 53, 1 (1979).

[6] E. Arimondo, in: Progress in Optics, Ed. E. Wolf, Vol. 35, Elsevier Science, Amsterdam 1996, p. 257.

[7] K.-J. Boller, A. Imamoglu, S.E. Harris, Phys. Rev. Lett. 66, 2593 (1991).

[8] J.E. Field, K.H. Hahn, S.E. Harris, Phys. Rev. Lett. 67, 3062 (1991).

[9] S.E. Harris, Physics Today 50, 36 (1997).

[10] S.E. Harris, J.E. Field, A. Kasapi, Phys. Rev. A 46, R29 (1992).

[11] A. Kasapi, M. Jain, G.Y. Yin, S.E. Harris, Phys. Rev. Lett. 74, 2447 (1995).

[12] O. Schmidt, R. Wynands, A. Hussein, D. Maschede, Phys. Rev. A 53, R27 (1996).

[13] L.V. Hau, S.E. Harris, Z. Dutton, C.H. Berhoozi, Nature 397, 594 (1999).

[14] M. Fleischhauer, M.D. Lukin, Phys. Rev. Lett. 84, 5094 (2000).

[15] C. Liu, Z. Dutton, C.H. Berhoozi, L.V. Hau, Nature 409, 490 (2001).

[16] D.F. Phillips, M. Fleischhauer, A. Mair, R.L. Walsworth, M.D. Lukin, Phys. Rev. Lett. 86, 783 (2001).

[17] M.D. Lukin, Rev. Mod. Phys. 75, 457 (2003).

[18] Z. Dutton, M. Budde, C. Slowe, L.V. Hau, Science 293, 663 (2001).

[19] C.H. van der Wal, M.D. Eisaman, A. Zibrov, A. Andre, D.F. Phillips, R.L. Walsworth, M.D. Lukin, Proc. SPIE 5115, 236 (2003).

[20] E. Cerbonesch, F. Renzoni, E. Arimondo, Opt. Commun. 208, 125 (2002).

[21] E. Cerboneschi, F. Renzoni, E. Arimondo, Opt. Commun. 204, 211 (2002).

[22] W. Franzen, Phys. Rev. 115, 850 (1959). 